\title{
TV/Series
}

$7 \mid 2015$

Le Pilote et la chute

\section{"An Englishman in New York »: actualisation, effets de Canon et transgressions dans Elementary de Robert Doherty (CBS, 2012-)}

Denis Mellier

\section{(2) OpenEdition}

Journals

Édition électronique

URL : http://journals.openedition.org/tvseries/278

DOI : 10.4000/tvseries.278

ISSN : 2266-0909

Éditeur

GRIC - Groupe de recherche Identités et Cultures

Référence électronique

Denis Mellier, « "An Englishman in New York »: actualisation, effets de Canon et transgressions dans Elementary de Robert Doherty (CBS, 2012-) », TV/Series [En ligne], 7 | 2015, mis en ligne le 01 juin 2015, consulté le 19 avril 2019. URL : http://journals.openedition.org/tvseries/278 ; DOI : 10.4000/ tvseries. 278

\section{cc) (1) $(9)$}

$T V /$ Series est mis à disposition selon les termes de la licence Creative Commons Attribution - Pas d'Utilisation Commerciale - Pas de Modification 4.0 International. 


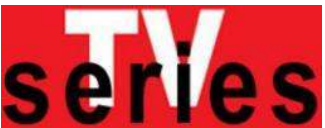

\title{
“An Englishman in New York": actualisation, effets de Canon et transgressions dans Elementary de Robert Doherty (CBS, 2012-)
}

\author{
Denis MELLIER
}

En constante expansion, la galaxie des réécritures apocryphes de Sherlock Holmes n'a cessé, depuis 1896, de produire des avatars acclimatant le détective de Baker Street à des lieux, des époques différentes, le confrontant, moins dans un processus d'adaptation que d'actualisation, à des formes sociales, idéologiques et technologiques renouvelées. Ces déplacements sont au principe de la série Elementary (CBS, 2012-) dans laquelle c'est à New York en 2010 que Holmes mène l'enquête, avec à ses côtés... Joan Watson ! L'actualisation procède-t-elle d'un effet de subversion des textes du Canon, rejouant sur un mode iconoclaste les formes conventionnelles du mythe afin de choquer les puristes, de décaper l'anglicité hautaine de l'original et de séduire les digital natives avec un Sherlock subversif, tatoué, sexuellement transgressif, et high tech ? Ou bien, le jeu est-il plus subtil et moins binaire, préférant tresser les valeurs imaginaires de la figure originelle avec une pratique intermédiale des fictions qui trouve dans les formes contemporaines la matière d'émancipations et de singularités, inversant alors la question de l'actualisation : au moyen d'un séduisant jeu de déplacements et de reformulations, Sherlock à NY n'apparaîtrait-il pas comme la figure métadiscursive susceptible de donner forme au conflit du contemporain et de l'épreuve virtuose de l'herméneutique. Question qui n'est pas sans pertinence en ces temps où les fictions policières télés semblent avoir délégué à l'assurance de l'expertise, du mentalisme et du profiling, et aux autres formes néopositivistes, l'exercice esthétique-logique de la pensée et de l'analytique comme virtuosité.

In constant expansion, the galaxy of Sherlock Holmes's apocryphal suites has never ceased, since 1896, to produce new avatars placing the Baker Street detective in new places and times, confronting him to various social, ideological and technological forms, in a process that is much less one of adaptation than one of actualization. Such move is the grounding principle of CBS's television series, Elementary (2012-), in which Sherlock is a consultant for the NYPD, living in Brooklyn in 2010 and sharing his Brownstone house with doctor... Joan Watson! Does this actualization proceed from an iconoclastic effect towards the canon, playing again the conventional formulas of the myth in a subversive mode in order to shock the purists, to scour the haughty Britishness of the original character, willing to seduce the digital natives with highly subversive, tattooed, sexually transgressive and high tech geek Holmes? Or is the game more subtle and less binary, weaving together the imaginary values of the original figure and an intermedia use of fictions finding in a contemporary context the ingredients for emancipation and singularities. Thus, one should reverse the question of actualization: by using a seductive game of moves and reformulations, Sherlock in NY may appear as a metadiscursive figure which could shape the conflictual relationships between contemporary visual culture and a virtuoso's hermeneutics test. Such a question may have particular relevance, in our times when TV series have abdicated to the authority of forensics, to mentalists or profilers, all those neo-positivist forms of thinking, the esthetico-logical sport of thought and a practise of analytics as sheer virtuosity. 
I could not bring myself to connive at the perpetuation of a hoax. Not only was there never a second Mrs. Watson; there was not even a first Mrs. Watson. Furthermore, there was no Doctor Watson.

Rex STOUT, « Watson was a woman » (1941)

$\mathrm{S}$ herlock Holmes n'est plus consultant pour Scotland Yard en 2012, c'est désormais au NYPD qu'il prête ses talents. De la terrasse où il a installé ses ruches pour le livre qu'il prépare, «Un manuel pratique d'apiculture assorti de remarques sur la ségrégation de la Reine " ${ }^{1}$, c'est le pont de Brooklyn que l'on peut voir. Même s'il retrouvera pour un épisode de la saison 2, le 221B Baker Street, pour l'occasion gentrifié par son frère Mycroft, c'est depuis une Brownstone qu'on croirait sortie d'un roman de Paul Auster qu'il exerce.

Exit l'incompétence légendaire de Lestrade, l'inspecteur Tobias Gregson est d'un tout autre niveau ${ }^{2}$. Aidan Quinn campe un personnage de flic irlandais, solide, efficace dont l'énergie et l'éthique sauront, à l'occasion, en remontrer à l'arrogance du limier britannique. Comme si le personnage qu'il interprétait dans Blink de Michael Apted en 1994 avait poursuivi sa carrière jusqu'à ce poste ; ou encore, comme si le lieutenant Kevin Sweeney qu'interprète Quinn de façon quasi synchrone dans Prime Suspect ${ }^{3}$, avait glissé d'une série à l'autre. Face au génie arrogant de Holmes, c'est un type qu'oppose le capitaine Gregson : le flic new yorkais. Les personnages canoniques 4 de la geste holmesienne font retour, ainsi de Moriarty, Watson, Mycroft, et l'on retrouvera Lestrade dans Elementary, moins comme un mauvais enquêteur qu'un plagiaire du talent de Holmes, sorte de loser médiatique qui, profitant du goût pour l'ombre propre à l'activité de conseil de Sherlock, retire devant les caméras tout le profit des enquêtes résolues5. Quant à John Watson? Une femme, Joan ${ }^{6}$ : ex-

'Elementary, 1.1, 16'45"'

${ }^{2}$ Tobias Gregson apparaît aux côtés de Lestrade dans « Une étude en rouge ». S’il remplit fonctionnellement le rôle de Lestrade, la nature de sa relation avec Holmes est très différente ; Holmes à Gregson [1.20, 9'50"] « [je souhaite] avoir le conseil avisé d'un grand enquêteur que je respecte et que j'admire ».

3 Prime suspect est une série américaine de 13 épisodes créée par Linda La Plante, diffusée sur NBC de septembre 2011 à janvier 2012. Cette série est une « adaptation » de la série britannique Prime Suspect (ITV) qui connut 7 saisons entre 1991 et 2006 et dans laquelle Helen Mirren interprétait l'inspecteur principal Jane Tennison, rôle repris dans la version américaine par Maria Bello.

${ }^{4}$ Les holmésiens désignent du terme de Canon, l'ensemble des 4 romans et des 56 nouvelles qui constituent le corpus des textes écrits par Arthur Conan Doyle.

52.16 [2'30"]

${ }^{6}$ Cette transgression-là aussi n'est pas récente ; Rex Stout avait publié en 1941 un classique des études holmésiennes, l'article « Watson was a woman » dans The Saturday Review of Literature, March 1, p. 3-4. Repris dans Profile by Gaslight, ed. E.W. Smith, New York, Simon \& Schuster, 1944. Par ailleurs, un téléfilm de 1987, The Return of Sherlock Holmes, réalisé par Kevin Connor, présentait aux côtés de Holmes (cryogénisé et ramené dans les années 80), une Jane Watson (descendante de John), interprétée par Margaret Colin. 
chirurgienne que le décès d'un patient a conduit à cesser son activité, elle gagne sa vie en accompagnant dans leur période postcure d'anciens toxicomanes. Recrutée par le père de Sherlock, elle est sa marraine chargée d'éviter les rechutes après sa désintoxication, loin des tentations londoniennes.

Écrite par Robert Doherty et diffusée sur CBS depuis 2012, la série Elementary compte à ce jour trois saisons chacune de 24 épisodes7, et repose sur l'actualisation de la figure de Sherlock Holmes. Il ne s'agit pas seulement de déplacer Holmes dans le temps mais de jouer sur la façon dont la transposition du personnage dans un contexte contemporain peut le déterminer nouvellement: les fondamentaux de sa méthode et certains traits de sa psychologie (arrogance, orgueil, etc.) sont intacts quand d'autres éléments relancent à nouveaux frais le profil du personnage. Certains ne sont qu'une mise à jour des savoirs et des moyens du détective - les nouvelles technologies n'ont aucun secret pour lui, il allie érudition livresque et googleïsation des connaissances, il lit à livre ouvert dans les entrailles d'un téléphone portable. D'autres représentent en revanche d'authentiques évolutions au regard de ses caractérisations conventionnelles : omniprésence comique de la sexualité, ouverture progressive au fil des deux saisons à l'empathie et à une compréhension d'autrui qui conduisent à substituer au seul intérêt dramatique de la résolution des intrigues, la fable continue et feuilletonnée de la relation entre Joan et Sherlock.

Ce personnage dont nombre de stéréotypes visuels ont été fixés dans les années 1940 par Basil Rathbone - casquette Deerstalker, pipe Calebasse, pèlerine ${ }^{8}-$ puis ramenés à une lecture plus littérale du contexte victorien dans l'interprétation qu'en a donné Jeremy Brett dans les années 1990, enquête, dans Elementary, au début du $\mathrm{xxI}^{\mathrm{e}}$ siècle ; Sherlock y porte des jeans, boutonne ses chemises jusqu'au col, n'a que faire de son apparence et arbore des tatouages plutôt rocks. Ils n'ont pas été faits pour la série, ce sont ceux de l'acteur Johnny Lee Miller qui incarne le détective. Goût du jour outrancier visant à dépoussiérer l'image canonique du détective? La coïncidence est finalement plaisante quand on songe à l'importance des motifs dermatologiques, cicatrices et tatouages dans les enquêtes du Canon'. Mais la série, au moins tout autant que sur l'actualisation temporelle, repose sur un déplacement culturel significatif. On a fait jouer, à l'ouverture de cette étude, certains des stéréotypes holmesiens et leur

7 Une quatrième saison est prévue pour 2015-2016.

${ }^{8}$ On se reportera par exemple à The Hound of Baskerville de Sidney Lanfield en 1939 ou The Adventures of Sherlock Holmes d'Alfred Werker, la même année. Il existe une abondante littérature holmesologique sur le caractère non-canonique de certains de ses attributs apportés à Holmes par sa postérité cinématographique.

${ }^{9}$ La dermatologie était une des spécialisations médicales du docteur Conan Doyle. 
recontextualisation new-yorkaise : un des symboles nationaux les plus marqués du monde britannique, parfaite expression d'une anglicité faite de victoriana, de cabs et de fog, lesté de toute une onomastique de signifiants et de notations de Paddington à King's Cross, de Mayfair à l'East End, arpente désormais Central Park et le Bowery ${ }^{10}$.

Le jeu de réécriture mené avec les conventions, leur persistance et leurs déplacements ainsi que la distanciation ludique présidant aux anamorphoses dont les éléments canoniques sont l'objet, constituent le fond même qui détermine le jeu du pilote -compris comme apparition, ou ici retour surprenant, lancement ou première fois. Mais ce jeu n'est pas sans incidence non plus sur l'élaboration, répétée d'épisode en épisode, de la chute - comprise comme clôture provisoire et relance de la fiction.

Quelle incidence le processus intertextuel de reprise apocryphe dans lequel s'inscrit pleinement Elementary - mais aussi d'une façon presque synchrone, la série britannique Sherlock ${ }^{11}$ - peut-il avoir sur les problèmes que posent les poétiques d'engendrement et d'ouverture (pilote) et celles qui supposent le jeu de clôture et de relance de la fiction dans le cadre d'une série dont la construction repose, en grande majorité, sur des épisodes bouclés (formula show ou série formulaire) ${ }^{12}$, à l'exception de quelques intrigues qui se déploient sur plusieurs épisodes et qui relèvent alors d'une structure modulaire ${ }^{13}$ ?

\section{L'univers apocryphe holmesien : une bibliothèque en amont du pilote.}

Mais ce jeu de déplacement et d'actualisation ainsi que le contenu parodique et réflexif ne doivent pas surprendre. La pratique est courante, depuis 1896, date d'un premier pastiche que l'on doit à Conan Doyle lui-même ouvrant ainsi le champ à toutes les réécritures à venir $^{14}$. Celles-ci courent de façon continue sur l'ensemble du $\mathrm{Xx}^{\mathrm{e}}$

\footnotetext{
${ }^{10}$ On trouverait, dans la galaxie apocryphe de ses réécritures, de nombreux exemples de «délocalisation » du personnage de Sherlock Holmes : ainsi Holmes, dans une perspective si ce n'est canonique du moins « classique ", s'est déjà rendu aux États-Unis, par exemple, dans un film de 1943, Sherlock Holmes in Washington de Roy William Neill.

${ }^{11}$ Écrite par Stephen Moffat et Mark Gattis, la série Sherlock est diffusée sur la BBC depuis juillet 2010 ; elle comporte, à ce jour, trois saisons de trois épisodes chacune.

${ }_{12}$ Nous reprenons ici les termes couramment employés dans les travaux sur les séries, et notamment proposés par Martin Winckler dans le glossaire de son livre, Les Miroirs obscurs, Paris, Au Diable Vauvert, 2005, à l'exception du terme « série-feuilleton » auquel nous préférons celui de série modulaire.

${ }_{13}$ C'est le cas dans Elementary des derniers épisodes de la saison 1 et des premiers de la saison 2, consacrés au traitement d'une variation anti-canonique impliquant Moriarty, Irène Adler et le Colonel Moran.

14 «The Field Bazar » est un pastiche que Conan Doyle écrit en 1896 pour une collecte de fonds destinée à l'Université d'Edimbourg. Voir Sherlock Holmes, the Published Apocrypha by Arthur Conan Doyle and Associated Hands, selected and edited by Jack Tracy, Boston, Houghton Mifflin Company, 1980.
} 
siècle, développant une galaxie apocryphe considérable qu'aucun autre personnage de fiction n'a quantitativement connue à ce jour. Les dimensions métalittéraires de ce jeu, les conceptions de la fiction et de ses usages qu'il implique, les porosités d'univers, méta et transfiction, tout comme les transmédiations ou les remédiations, changements de supports et de formes médiatiques ${ }^{15}$ qu'ont connus les divers avatars de Holmes, ne sont pas ici directement mon objet ${ }^{16}$, même si Elementary, tout comme la série britannique synchrone, Sherlock, en procèdent toutes deux et en prolongent l'esprit. Pas plus mon souci, les porosités fantasmatiques ou ludiques qui, chaque année, envoient en pèlerinage à Reichenbach les membres des sociétés holmésiennes, incarnant tel ou tel personnage du corpus holmésien en costume d'époque victorien... ici le Colonel Moran, là Irène Adler.

Qu'il suffise aux néophytes de savoir qu'à l'emplacement supposé du 221B Baker Street se trouve le siège londonien de l'Abbey National, une compagnie bancaire qui appointe un secrétaire pour répondre au courrier que reçoit le détective, et que les éditions Penguin avait même publié dans les années 1980 une anthologie de cette correspondance $^{17}$. Jeu d'actualisation ${ }^{18}$ de la fiction, mais aussi jeu de lecture et d'écriture, il peut être lu comme sommet de l'illusion réaliste, vertige de la métalepse ontologique ou pouvoir ludique de la fiction. Quant à ceux qui découvriraient un tel univers, ils constateront vite qu'il n'est pas une grande figure fictionnelle ou référentielle qui ne puisse entrer en relation fictionnelle avec celle de Sherlock Holmes : Freud, Marx, Dracula, Dorian Gray, Stevenson, Sarah Bernhardt, Catulle Mendès, Oudini, etc. Quant aux projections sur lesquelles reposent Sherlock et Elementary, les inventions uchroniques et rétrofuturistes du steampunk ont fait autrement voyager Holmes ${ }^{19}$ et au rayon des anachronismes jubilatoires, René Réouven a déjà renvoyé Holmes prêter main forte à Dupin, séchant sur le double assassinat de la rue Morgue, grâce à la machine à remonter le temps empruntée pour l'occasion à Wells ${ }^{20}$. Stephen Moffat, le scénariste de Sherlock, rappelle que l'idée de déplacer Holmes dans l'actualité de son public était déjà au principe des films avec Basil Rathbone et Nigel Bruce situant

\footnotetext{
15 Sur les adaptations cinématographiques et télévisuelles, voir Alan Barnes, Sherlock Holmes on Screen, The Complete Film and TV History, Londres, Titan Books, 2011 ; Max Philippe Morel, Sherlock Holmes au cinéma et à la télévision, Strasbourg, Les Évadés de Dartmoor, 2013.

${ }^{16}$ Pour un traitement développé de ces questions, voir notre article « L'Aventure de la faille apocryphe ou Reichenbach et la Sherlock-fiction », in Sherlock Holmes et le signe de la fiction, éd. Denis Mellier, Fontenay Saint-Cloud, coll. "Signes », ENS éditions, 1999, p.135187.

${ }^{17}$ Letters to Sherlock Holmes, éd. Richard Lancelyn Green, Londres, Penguin books, 1985.

${ }^{18}$ Dans cette occurrence seulement, actualisation est pris non dans son sens temporel mais dans celui de réalisation, comme dans l'opposition anglo-saxonne actual/fictional.

${ }^{19}$ Voir, par exemple, Thomas Day, L'instinct de l'équarisseur : vie et mort de Sherlock Holmes (Mnémos, 2002), Paris, coll. « Folio SF », Gallimard, 2004.
}

${ }^{20}$ René Réouven, Histoires secrètes de Sherlock Holmes, Denoël, 1993. 
l'action durant les années 1940, celles de leur production, et qui s'ouvraient à l'actualité en abordant le conflit avec l'Allemagne et les activités d'espionnage ${ }^{21}$. Et plus loin encore sur la flèche du temps, la série d'animation Sherlock Holmes au XXII ${ }^{e}$ siècle diffusée sur Fox News de 1991 à 2001.

Loin d'être étonnantes, ces " adaptations » actualisantes sont à resituer dans un processus apocryphe continu dont elles sont une expression contemporaine. Ce processus témoigne de la plasticité imaginaire de la figure de Sherlock Holmes et du personnel qui l'entoure (Watson, Lestrade, Mycroft, Irène Adler, Moriarty) ainsi que de l'efficace et de la séduction toujours active des expansions fictionnelles de son mythe. Ces expansions ont très tôt suggéré un jeu de lecture s'émancipant des limites d'une mimésis ou d'un simple ancrage générique réaliste et conventionnel. Le jeu repose sur une circulation des figures et des fictions, il élabore ses propres règles et conditions de possibilité. Replacé dans une perspective réflexive et transtextualiste ${ }^{22}$, le jeu de réécriture qui s'effectue depuis le Canon holmésien suppose une circulation plus ou moins informée, selon les lecteurs, entre la matrice hypotextuelle, ses transformations hypertextuelles et ses excroissances apocryphes. Elles correspondent, dans Sherlock comme dans Elementary, à la reprise de matériaux anciens empruntés, avec distanciation et humour, aux textes de Conan Doyle - des noms, des fragments d'intrigue, des répliques fameuses, des tics et des comportements notoires. Ces divers éléments se voient alors déplacés, travestis ou réinvestis de dimensions plus ou moins neuves ou autonomes : c'est en ce sens que ces deux séries ne sont pas, à proprement parler, des adaptations qui «dépoussièreraient », aux conditions du présent, les intrigues anciennes remises au goût du jour. Il faut, à certains égards, être un holmésien déjà assez informé des textes du Canon, ou de leurs adaptations dans la série diffusée par Granada avec Jeremy Brett par exemple, pour retrouver les éléments indiciaires précis rattachant directement les avatars de Elementary à leurs hypotextes ; d'autant que, dans le cas de Sherlock, les épisodes d'une durée double (90') de ceux d'Elementary (42') sont construits à partir d'emprunts à plusieurs aventures du détective tressées entre elles ${ }^{23}$. Si les identités, les correspondances, voire une forme de fidélité à l'esprit sous l'anamorphose contemporaine déterminent directement

\footnotetext{
$21 \quad$ Voir l'entretien avec Stephen Moffat sur http://www.lemonde.fr/culture/article/2014/o4/18/sherlock-holmes-est-comme-james22 J'emploie ici ce terme et ceux qui en découlent (hypo/hypertexte), dans les limites strictes de la caractérisation qu'en donne Gérard Genette dans Palimpseste, la littérature au second degré, Paris, coll. « Poétique », Seuil, 1982.

${ }^{23}$ Les épisodes 2 et 3 de la première saison de Sherlock sont par exemple construits avec des éléments empruntés à Une étude en rouge, à La Vallée de la peur mais aussi à Les Cinq pépins d'orange.
} 
les projets des scénaristes, le large succès de ces deux séries ${ }^{24}$ montre que ce jeu d'érudition n'est cependant pas - comme pour tout hypertexte - une condition nécessaire à la compréhension de la série et au plaisir qu'en tire le public. En revanche, une fois notée, et cela dès les retrouvailles que propose le pilote, cette circulation de motifs, de caractères et de propriétés originales offre au spectateur le plaisir spécifique des écritures parodiques, des allusions et des effets de connivence qu'autorise la bonne mesure des déplacements et des origines reconnues.

Elementary propose une manière d'index du corpus canonique constitué de faux-amis, ce qui contribue à faire émerger alors l'autonomie de ses propres intrigues et à désengager l'avatar de l'autorité du Canon. Cette émancipation est d'autant mieux marquée que, par les effets d'onomastique et les traits imaginaires que lui emprunte Doherty, le Sherlock Holmes new-yorkais tire sa consistance d'un univers textuel et fictionnel antérieur - bien connu ou non du spectateur -, mais qui fait retour sous la forme d'échos et d'allusions. Les exemples sont trop nombreux pour une analyse serrée du processus ou un large relevé des items. Le travail sur l'onomastique des personnages est significatif d'un univers qui ne s'actualise pas par l'exactitude d'une transposition terme à terme, mais qui se construit sur des identités déterminées mais incomplètes et déplacées, composites et en attente d'une incarnation spécifique dans la fiction new-yorkaise.

Dans 1.19, une amie de Holmes, helléniste émérite, trouve refuge chez lui après une rupture douloureuse. Obsessionnelle du rangement, elle supporte mal le désordre de Holmes. Ils conviennent qu'elle repassera une fois par semaine pour le ménage : elle se nomme Mlle Hudson. Dans 1.22, le kidnappeur d'Irène Adler s'appelle Stapleton qui est le nom du coupable dans Le Chien des Baskerville; l'argument de l'épisode 1.17 (Savant Fou/Possibility Two) n'est pas construit sur celui de The Adventure of The Musgrave Ritual, alors qu'un des personnages principaux se nomme Musgrave, etc.

S'il est systématique, le procédé allusif n'appelle pourtant aucun effet de correspondance stricte entre le Canon et l'actualisation ; si elle est repérée, l'allusion fait constater la liberté avec laquelle le matériau originaire est redistribué pour créer des intrigues singulières. Exemplaire de l'autonomisation de cet univers auto-référentiel, l'utilisation du nom de Newgate pour désigner la prison où sont envoyés les criminels dans Elementary. Newgate fut la première prison new-yorkaise, mais elle ferma en 1828 au profit de Sing Sing. La

${ }^{24} \mathrm{La}$ consultation des audiences sur les sites dévolus à ces mesures montre que l'une comme l'autre série sont très largement regardées aux États-Unis, en Grande Bretagne ou en France. Voir http://www.audiencesusa.com/ ; http://www.leblogtvnews.com/articlebilan-d-audience-de-la-saison-1-d-elementary-sur-m6-122772623.html (dernière consultation : 15 juin 2015). 
prison de Newgate à Londres ferma, elle, en 1902 et c'est sa valeur imaginaire qui veut, par exemple, que dans les aventures de Harry Dickson, écrites par Jean Ray et au départ un apocryphe holmésien ${ }^{25}$, Newgate demeure la référence criminelle majeure, celle que l'on trouve chez Dickens et Conan Doyle. De la même façon, le monde d'Elementary est moins un monde mimétique construit strictement sur les seules realia du New York des années 2010 qu'il ne fonde sa consistance sur une vaste bibliothèque de signifiants, de signes, de symboles et de figures imaginaires. Tous créent comme des brèches mémorielles et poétiques dans l'impeccable bouclage narratif des enquêtes. Ces figures s'entendent d'autant plus nettement qu'elles n'ont pas de valeurs logiques ou indiciaires particulières, qu'elles semblent bien plus gratuites que fonctionnelles et qu'elles ouvrent un mode de lecture de la fiction d'Elementary émancipé des seules obligations policières de la résolution.

Les épisodes empruntent finalement assez peu de traits aux intrigues originales inventées par Conan Doyle et n'en sont que d'assez lâches variations. Dans Elementary, le mythe holmesien se voit confronté aux formes criminelles produites par la société américaine, son mode de vie et ses formes d'imaginaire de la violence et de la mort : c'est particulièrement vrai des motifs du kidnapping et surtout du serial killer. A plusieurs occasions, les déductions de Holmes l'amènent à conclure que le meurtre n'est que le premier d'une série qu'il ouvre ou qu'il poursuit, retrouvant là un motif commun des fictions policières contemporaines dans lesquelles il faut souvent que la perspicacité de l'enquêteur impose à ses collègues l'évidence de la série que, seul, il comprend. De tels effets de circulation entre les formes canoniques, l'architextualité du récit policier contemporain et les transgressions hétérodoxes, perçues ou inaperçues, dépendent de la motivation des lectures - reconnaissances et interprétations des relations - que les spectateurs sont à même d'activer à partir de la proposition faite par le pilote et au-delà, que vont décliner et relancer à chaque fois les épisodes.

Érudition holmésienne jouant avec un corpus considérable, stéréotypie minimale et partagée, compétence générique policière interprétant la figure de Holmes à l'intérieur du champ des séries policières criminelles : l'effet d'ouverture et de séduction sur lequel parie le pilote d'une nouvelle série est, pour Elementary, déterminé par la mesure très variable que le spectateur peut effectuer d'un matériau dont le caractère demeure, dans l'usage qu'en fait Doherty, volontairement allusif et imprécis : ce régime allusif n'entend pas renvoyer à des objets exacts, mais plutôt jouer de leur puissance d'évocation dans le cadre d'un corpus imaginaire bien plus vaste, le

${ }^{25}$ Voir sur les relations de Sherlock Holmes et de Harry Dickson notre article cité précédemment note 16 . 
Canon et au-delà, l'univers apocryphe tout entier. La première fois que John Watson rencontre Holmes, dans Une étude en rouge (1887), ce dernier est en train de rouer de coups de canne un cadavre afin d'étudier la formation des hématomes. La première fois que Joan Watson rencontre Holmes, dans le pilote de la série, une prostituée sort de chez lui, un gros plan sur les barreaux d'une échelle montre deux menottes. Facilité iconoclaste ou rétrolecture à partir d'une focale sado-maso, d'une variation érotique qui renvoie vers un impensé victorien, cette fameuse divided-self ${ }^{26}$ dont Holmes, tout autant que Jekyll ou Dorian Gray, aurait été l'expression?

Parce que l'antériorité du mythe holmésien est si prégnante, tout comme sont considérables les nuances amenées par la somme de ses avatars, parce que le projet d'Elementary est insécable, à quelque degré que ce soit, de sa matière canonique, si ce n'est nécessairement dans la réception du spectateur tout au moins dans le travail d'adaptation et de déplacement du scénariste, le pilote ne peut qu'introduire à une nouveauté conditionnelle. Si tout pilote escompte, idéalement, ses effets de l'apparition d'un objet neuf, dans le cas d'Elementary l'antériorité des corpus et la multiplicité des degrés de réalisation des allusions sont telles que ses effets sont inévitablement stratifiés : loin d'être un défaut de la série, cela témoigne plutôt de la pluralité de lectures qu'elle permet d'accueillir selon les différences de positions et de savoirs spectatoriels.

\section{2. «An Englishman in New York »: d'une dualité surjouée}

Ce n'est donc peut-être pas tant le procédé de l'actualisation temporelle du personnage qui est l'argument scénaristique déterminant d'Elementary que la décontextualisation sur laquelle repose la série, c'est-à-dire l'américanisation de l'univers intellectuel attaché à la détection holmesienne. L'effet de distanciation comique de la série joue de cette confrontation entre des univers policiers archétypaux dont l'opposition constitue le paradigme dominant sur lequel s'est écrite l'histoire du roman policier : modèle de la détection à l'anglaise contre imaginaire réaliste du hard boiled, avec son univers criminel urbain, violent, né avec le roman noir dans les années 30. C'est Sherlock contre Marlowe, Agatha Christie contre Hammett, le jeu logique contre le néonaturalisme du roman criminel, etc. Au principe du tissu ludique d'Elementary, ce dualisme des formes policières sert aussi à développer des motifs singuliers qui ont notamment trait à l'évolution des personnages, à la recharge de la fiction et au statut de la parole $^{27}$.

\footnotetext{
${ }^{26}$ Sur la thèse classique de la schize victorienne, voir Masao Miyoshi, The Divided Self: a Perspective on the Literature of the Victorians, New York, University Press, 1969.

${ }^{27}$ Voir les deux dernières parties de notre étude.
} 
Cependant, l'effet de cette dualité peut s'envisager d'une autre façon: dans l'imaginaire contemporain, l'articulation des formes britannique et américaine a déjà eu lieu à diverses occasions. Certaines fictions explorent une sorte de victorianisation de l'enquête américaine, traitant le motif de l'émergence de la police new-yorkaise selon des formes très inspirées de la mythologie criminelle victorienne et usant de mythèmes communs, comme les bas-fonds et les sociétés secrètes. On peut établir un corpus retro-dix-neuvièmiste multipliant les interactions imaginaires entre les lieux et les tropes: Amérique/Angleterre ; Londres/New York; enquête policière/ouverture fantastique ; roman d'aventure/thriller d'action avec l'implantation de la figure du tueur en série, venue de l'origine anglaise de Jack l'Eventreur mais développée à partir de la prégnance de la figure du serial killer dans la culture de masse nord-américaine de la fin du $x^{\mathrm{e}}$ siècle. Des romans comme L'aliéniste de Caleb Carr, le Waterworks de Doctorow, dont l'éditeur de la traduction française ne manqua pas d'évoquer sur sa quatrième de couverture l'imaginaire holmésien du roman ${ }^{28}$, aujourd'hui des séries télé comme Copper une unité de policiers dans le New York de la guerre de Sécession - et Ripper Street ${ }^{29}$ - une unité de policiers dans le Whitechapel des années 1890 - témoignent de l'intense circulation de motifs qui s'effectue à partir d'un imaginaire de type syncrétique. Dans Ripper Street, la situation du consultant, dans le Scotland Yard de 1890, représente même le chiasme parfait de celle d'Elementary: comme une manière de Holmes inversé, c'est un médecin légiste, rationaliste, athée, mais surtout américain qui amène dans l'East End le savoir et l'énergie d'un monde nouveau dans une société ancienne qui affronte les conditions de sa modernité.

Ce syncrétisme imaginaire et fictionnel montre que la question de l'actualisation ne peut être simplement comprise comme la mise au présent d'un matériau ancien : on en resterait alors à une des logiques de l'adaptation ou du remake consistant à décontextualiser et à recontextualiser une fiction par la modification de son lieu, de son époque et de certaines des conditions d'identification essentielles de ses personnages ou de son intrigue - ici, par exemple, l'ouverture du récit de la performance logique aux formes de la violence et du gore visuels caractéristiques de la norme contemporaine en matière de réalisme criminel ${ }^{30}$. Une des conséquences de l'actualisation est la

${ }^{28}$ Caleb Carr, L'aliéniste (1996), Paris, Pocket, 2004 ; E.L. Doctorow, Waterworks (1994), La Machine d'eau de Manhattan, Paris, Flammarion, 1996.

${ }_{29}^{2}$ Ripper Street est une série britannique de Richard Barlow dont les deux premières saisons ont été diffusées sur BBC one en 2012 et 2013. Copper est une série américaine de Tom Fontana dont les deux premières saisons ont été diffusées sur BBC America en 2012 et 2013.

$3^{0}$ Voir l'épisode [1.20; 31'], Fiel, Mensonge et Vidéo/Dead Man's Switch, la scène de découverte du cadavre semble être clairement démarquée de celles de Seven (Bryan Fincher, 1995) (découverte des victimes des péchés de gourmandise et de fainéantise). 
systématisation du crime de sang dans toutes les intrigues de Elementary, étalonnant la fiction policière du Canon sur les réquisits des séries policières contemporaines. Très peu d'histoires du Canon sont en fait liées à des meurtres, et le ressort criminel des intrigues de Conan Doyle - ce qui peut surprendre un spectateur de séries policières contemporaines - relève de l'extorsion, de la séquestration, de l'espionnage, de menaces et de chantages. Uri Eisenzweig a fait remarquer que la nécessité narrative du cadavre silencieux, garant d'un récit à reconstituer par l'enquête, s'imposera dans le récit de détection après le modèle holmésien et notamment avec l'enquête telle qu'elle se développera dans les récits policiers d'Agatha Christie ${ }^{31}$.

L'actualisation ne relève-t-elle pas que de façon superficielle de la question du contexte, pour toucher en profondeur à la forme esthétique et à ses implications idéologiques ? Que Sherlock utilise les technologies de son époque répond à une exigence de réalisme. En revanche, que cet usage ait une incidence sur la forme de l'image ou sur le dispositif de vision représenté dans la série constitue une actualisation sûrement plus significative de ce que notre époque exprime d'elle-même à travers la figure de Holmes. Dans la série Sherlock, les incrustations numériques dans l'image représentent la façon dont fonctionne l'univers mental du détective, elles constituent une manière visuelle d'accès à son processus intellectuel, tout comme les ralentis, la voix off et la construction proleptique anticipent les combats de Sherlock Holmes dans les adaptations cinématographiques de Guy Ritchie. Dans Sherlock, l'image semble désormais conçue comme une surface d'inscription des données digitales, indifférenciant les sources - écrans d'ordinateurs, téléphones portables, caméras de surveillance. Dans Elementary, la première performance de Holmes il fait une déclaration enflammée à Joan qui est en fait l'imitation parfaite de celle que joue à la télévision un acteur - s'effectue devant un dispositif de sept écrans diffusant des programmes différents que Sherlock suit simultanément. Actualisation des thèmes et des motifs, actualisation formelle, actualisation des discours : laquelle, dans ces visions contemporaines du personnage est-elle de nature à faire davantage crier au scandale et à la transgression les agents de l'orthodoxie holmesienne?

Sur ce point, une précision s'impose. Jusqu'ici, le terme " holmesien » a été employé de façon générale et il est, à ce stade, nécessaire d'effectuer une distinction entre "holmesiens " (holmesians) et «sherlockiens » (sherlockians) : les premiers sont les garants de la cohérence d'un corpus centré sur le seul Canon et les hypertextes qui en découlent de façon vraisemblable et chronologiquement motivée. Leurs travaux construisent la continuité

${ }^{31}$ Uri Eisenzweig, Le Récit impossible forme et sens du roman policier, Paris, Bourgois, 1986. 
parfaite de la geste holmesienne, précisant la datation des aventures, résolvant les hiatus qui pourraient se présenter entre le monde référentiel et l'univers décrit par Arthur Conan Doyle. Les holmesiens ont eux-mêmes à la main un horaire ferroviaire Bradshaw et vérifient dans la presse d'époque les conditions météorologiques afin de dater au plus juste telle ou telle aventure. Les " sherlockiens », eux, sont des fictionalistes, des borgesiens qui pratiquent la bifurcation et qui s'autorisent tout type de réécriture, explorant le potentiel imaginaire des expansions fictionnelles.

Les effets de transgression qu'introduiraient les actualisations contemporaines de Sherlock Holmes sont donc à relativiser selon la nature du jeu fictionnel dont un lecteur, ou une communauté interprétative de lecteurs, crédite l'univers holmesien. Également, comme on l'a déjà souligné, parce qu'ils s'inscrivent dans une longue tradition de pratiques apocryphe et parodique, il ne manque pas de volumes conservés dans l'Enfer du British Museum autrement plus transgressifs quant aux mœurs de Holmes, de Watson et de Mrs Hudson que ce pilote rapportant l'installation sous le même toit de Joan Watson ou les relations tarifées de Sherlock. Et enfin, parce que les ruptures et les innovations ne sont pas toujours forcément là où elles semblent s'inscrire de la façon la plus voyante.

J'ai bien sûr ici conscience d'avoir choisi une approche critique effrontément sherlockienne. La vérité m’oblige à signaler que les ayants droits américains de Conan Doyle ont, par exemple, menacé de s'opposer au tournage du second film de Guy Ritchie, si son acteur Robert Downey Jr et ses scénaristes persistaient à laisser entendre l'homosexualité de Holmes. L'orthodoxie fixe elle-même les limites où commence le scandale des actualisations et des jeux de fictions. Ainsi l'auteur britannique d'apocryphes canoniques ${ }^{32}$, June Thompson écritelle : " [the Holmes biographies] irritated me because they were made up and had Holmes doing all manner of fantastic things like travelling to America as an actor and one even questioned his sexuality. This is so unnecessary. To my mind there is enough information in the Canon not to need to invent new details ».33

L'effet d'une transgression se mesure moins à la nature du geste qui l'opère qu'à la perspective de celui qui la perçoit et à sa conception de la fiction.

\section{Enjeux et paradoxes des actualisations}

Une des logiques d'institutionnalisation et de légitimation critique de la paralittérature, de la culture de masse et de la culture pop

\footnotetext{
32 Voir June Thompson, Holmes and Watson : a Study in Friendship (1995), Paris, coll. « Le Masque » ${ }^{\circ}{ }^{2} 292$, Librairie des Champs-Élysées, 1996.

${ }^{33}$ June Thompson, « Book Review », Sherlock Holmes Gazette, nº 12, summer 1995, p.28.
} 
- termes employés ici dans leur dimension pragmatique et sans aucun arrière-plan axiologique - consiste à reconnaître dans les fictions une dimension réflexive - assez évidente depuis Poe en ce qui concerne le roman policier. Cela permet notamment de lire la répétition formulaire comme une poétique de la variation, conduisant au dépassement des contraintes génériques en leur subversion. Or, le terme est trop galvaudé car, en son principe, la subversion est offensive et vise à détruire la forme qu'elle reprend pour la défaire. Ni Sherlock ni Elementary n'entendent subvertir la formule narrative et sémiologique caractéristique du modèle holmesien. John a beau être devenu Joan, elle reste une «Watson », débarrassée, certes, de la régie narrative que lui assignait «l'effet Watson »34, mis au point par Conan Doyle, mais elle n'est demeure pas moins placée dans sa fonction classique d'adjuvante à l'enquête, en tout cas, dans les premiers épisodes de la saison 1.

Dès lors, les éléments d'actualisation les plus tapageurs (sexe, drogue, anticonformisme radical, mépris des conventions sociales ici largement accentué par rapport aux attitudes dandy ou bohème du Holmes original, etc.) constituent-ils d'authentiques transgressions? Ils semblent, à l'examen, plus superficiels que décisifs, et surtout plus fonctionnels que critiques. Sans contester l'orthodoxie d'un imaginaire que la relecture contemporaine jugerait dépassé ou contestable, ces éléments confortent le mécanisme scénaristique de la série, les résolutions et les méthodes hypothético-déductives de Holmes.

Il semblerait, par ailleurs, que dans le match des satisfecit holmesiens quant au meilleur dosage d'orthodoxie et de mise à jour, l'avantage aille très souvent à la série britannique. L'interprétation que Benedict Cumberbatch offre de Holmes au XXI ${ }^{\mathrm{e}}$ siècle l'inscrit, assez nettement, dans la lignée de celle, déterminante pour les relectures contemporaines du personnage, campée par Jeremy Brett dans la série d'adaptations de Sherlock Holmes tournée par Granada Television et diffusée entre 1984 et 1994. L'argument quant à cette préférence n'est pas étayé par une étude quantitative rigoureuse ni même par la lecture assidue de forums de fans des deux séries : tout au plus, elle provient empiriquement d'échanges avec des holmésiens de divers degrés, de l'amateur occasionnel au connaisseur éclairé, du sérievore à l'anglophile attaché à l'imaginaire victorien, du fan de polars US à l'amateur de steampunk, ouvert à tout réemploi transfictionnel des victorianas.

On écartera, comme seule explication de cette préférence relative, ce que pourrait avoir d'outrancièrement transgressif l'américanisation ainsi que la féminisation opérés par Robert Doherty dans le scénario de la série. On imagine bien qu'un holmesien soit ici moins ouvert à de tels jeux qu'un sherlockien prêt à plus de souplesse

${ }^{34}$ Daniel Couégnas, « L'effet Watson », Les cahiers de l’imaginaire, $\mathrm{n}^{\circ} 14$. 
avec les données constitutives du Canon. Mais y a-t-il davantage "scandal in orthodoxia » à faire de Holmes un ex-junkie en cure post rehab, bardé de tatouages, aux pratiques sexuelles pimentées, que d'assister à sa marvelisation sous l'impulsion conjointe de Guy Ritchie et de Robert «Iron Man » Downey Jr. dans les deux long-métrages Sherlock Holmes (2009) et Sherlock Holmes : jeu d'ombres (2011)?

Si les spectateurs avaient découvert avec le Young Sherlock Holmes (La Pyramide de feu) de Barry Levinson en 1985, un Sherlock Holmes au pays d'Indiana Jones, c'est à une véritable cartoonisation que se livrent les deux films de Ritchie: c'est dans l'esprit des blockbusters américains actuels et des effets spéciaux numériques qui caractérisent les récentes adaptations des comics et des romans graphiques que se situe le projet d'interprétation de Ritchie. À l'origine, c'est un comic non publié de Lionel Wigram et non les textes du Canon. Selon Wigram, sa lecture se veut proche du texte original, notamment en insistant sur le côté bohême du personnage ou sa pratique du bartitsu. Effectivement mentionné dans les textes, cet art martial 35 permet surtout à la mise en scène de multiplier des scènes de combat tout à fait calquées sur celles qu'offre le cinéma d'action contemporain. Faisant la promotion du film lors du Comic-Con ${ }^{36}$ de 2009, l'acteur Robert Downey Jr déclarait envisager Sherlock Holmes comme un super-héros ${ }^{37}$ et c'est donc littéralement que son Sherlock Holmes doit être vu en reflet de son personnage d'Iron Man. Mais, à nouveau, l'effet de l'actualisation est peut-être ici moins sémantique que formel : si l'imaginaire holmesien s'en trouve modifié, c'est parce que la forme cinématographique qu'adopte Ritchie est en elle-même porteuse d'un imaginaire visuel, médiatique et numérique qui se saisit du mythe holmésien, du film à costume revendiqué par son scénariste avec sa date victorienne de 1891. Transgression iconoclaste ? Renouvellement visuel bienvenu? Adaptation du mythe holmesien aux normes de consommation visuelle contemporaine? Au-delà de l'appréciation, c'est l'hétérogénéité des imaginaires qui réalise le plus exactement l'actualisation formelle.

Dans Elementary, l'actualisation a pour effet immédiat de confronter une figure surdéterminée par sa tradition imaginaire et un contexte, qui l'est au moins tout autant, par son omniprésence

\footnotetext{
35 Voir la très instructive, et très holmesologique, notice «bartitsu » (http://fr.wikipedia.org/wiki/Bartitsu [dernière consultation : 15 juin 2015]).

${ }^{36}$ Le Comic-Con International de San Diego est une grande convention qui se tient l'été depuis 1970. D'abord consacrée à la bande dessinée, elle s'est progressivement ouverte à de larges domaines de la culture pop : cinéma, séries télés, animes et mangas, jouets ou jeux vidéo (http://www.comic-con.org/ [dernière consultation : 15 juin 2015]).

${ }^{37}$ Sur cette hypothèse de la dimension superhéroïque du personnage, voir Natacha Levet, Sherlock Holmes, de Baker Street au grand écran, Paris, Autrement, 2012 et Catherine Truchon, "Sherlock Holmes ou le surhomme détective », http://popenstock.ca/dossier/article/sherlock-holmes-ou-le-surhomme-detective (dernière consultation : 15 juin 2015).
} 
médiatique et culturelle : la tradition britannique de la détection et le monde américain de la série policière. Baker Street vs Hill Street Blues; Sherlock Holmes vs n'importe quelle série policière télévisée déclinant les formes de la criminalité urbaine dans une métropole nord-américaine et centrant son action sur son personnel policier : enquêteurs récurrents, flic ou détective solitaire, tandem de profilers et de policiers, buddy series, équipe de policiers, de légistes, d'experts, et toutes les combinaisons possibles en découlant.

Mais le mythe holmesien excède sa matière policière et ne réside pas uniquement dans son imaginaire logique, son anglicité, sa scénographie faite de coups de théâtre déductifs ; et c'est dans cette perspective que le processus d'actualisation dans Elementary s'avère particulièrement intéressant. Pour bien des spectateurs gavés ad nauseam des démonstrations imparables fournies par l'autorité de la grande machine néopositiviste des séries à argument médico-légal (des Experts [CBS, 2000-] à NCIS [CBS, 2003-]) ou à certitude psychocognito-comportementaliste (de The Mentalist [CBS, 2008-2015] à Lie to $\mathrm{Me}$ [FOX, 2009-2011] $)^{38}$, le mythe holmesien est celui d'une relation intellectuelle, peut-être dissymétrique sur le plan des talents entre Holmes et Watson mais qui devient l'expression d'une amitié absolument essentielle à l'argument des fictions. La nature et la qualité de ce lien constituent le ressort de bien des réécritures. Si le thème de l'amitié est central, dans Sherlock comme dans Elementary, c'est cette seconde série qui en pousse l'exploration scénaristique le plus avant : pas seulement parce que Watson y est une femme et que peut se poser la possibilité d'une évolution de leur relation vers le registre sentimental, mais parce que Sherlock y fait l'expérience de la transmission de son savoir, que la question de sa pathologie est explicitement et continûment thématisée au fil des épisodes et que l'action de Watson dans son traitement est jugée, par Sherlock luimême, déterminante; enfin, que la question de l'émancipation de Watson devient un des éléments scénaristiques moteurs de la série. Elementary tire sa dynamique de la tension entre le jeu formulaire de clôture des intrigues et une ligne de force continue, qui substitue au récit de la génialité solipsiste celui d'une parité qui s'invente.

\section{Drogue, soin, transmission : dynamique de la chute}

La parité peut s'entendre de deux façons. Tout d'abord, comme l'expression d'une demande du monde social et culturel dans lequel se déroule l'action : en ce sens, une lecture culturaliste des composantes idéologiques et sociologiques de la série s'impose aisément. Joan

${ }^{38}$ Voir sur ces questions la thèse de Maud Desmet, Les Confessions silencieuses du cadavre : de la fiction d'autopsie aux figures des morts dans les séries et films policiers contemporains (1991-2013), soutenue en mars 2013 à l'université de Poitiers. 
Watson, dans ses valeurs et ses discours, est l'incarnation d'une perspective libérale, éthique, post-féministe, tournée vers les bonnes pratiques, respectueuse d'autrui et de l'environnement. C'est même un des ressorts comiques de la série que de voir comment la radicalité et la morgue de Sherlock Holmes heurtent profondément chez Watson ce qu'un point de vue européen se plaît souvent à désigner chez les américains comme de «bons sentiments ». La virtuosité cynique, le goût du paradoxe et la théâtralité sont des travers européens et aristocratiques qui ne manquent de choquer le pragmatisme démocratique - et petit bourgeois, bobo, si l'on veut - de Joan. Le choix de l'actrice Lucy Liu, d'origine chinoise, et de l'adjoint principal de Gregson, l'enquêteur Marcus Bell joué par un acteur noir, Jon Michael Hill 39 , témoigne d'un souci de représentation du multiculturalisme new-yorkais. La série navigue alors avec habileté dans les divers types ethniques et sociaux que lui offre la métropole, faisant son miel de la confrontation d'un Sherlock survolté avec toute une palette de stéréotypes sociétaux, de la galeriste branchée aux executive women, des avocats d'affaire aux traders de Wall Street, du philistinisme néo-libéral à l'inculture au quotidien.

Au-delà de cette première acception de la parité, comprise comme équilibre ou équité dans les représentations sociales et ethniques, sexuelles et identitaires, il en est une autre qui correspond à la prise de conscience par Sherlock, après le traumatisme de la perte d'Irène, de la nécessité d'un pair garant d'une relation dont il fait dépendre sa stabilité psychique même :

Q. Qu'est-ce qui vous pousserait le plus à rechuter ?

SHERLOCK. Je n'ai pas de pair. Aucun qui soit sain, en tout cas... ce qui revient à ne pas en avoir. Je ne peux vraiment discuter qu'avec un nonpair. Donc avec n'importe qui. J'ai fait des progrès, bien sûr, mais... je ne sais pas si je peux en faire encore beaucoup. Si je ne peux jamais apprécier vraiment une relation, quand vais-je arrêter d'essayer d'en entretenir?

Q. Vous n'avez pas encore renoncé au monde?

SHERLOCK. Mais je n'ai pas de pair. Voilà ce qui pourrait me pousser à rechuter. [2.21, 00'40"].

Depuis le Canon, l'ennui existentiel affectant Holmes permettait de lier inactivité intellectuelle et recours à la drogue. Dans Elementary, la passion de l'énigme pour elle-même laisse progressivement place à une interprétation de l'addiction comme pure compensation au manque de l'autre : perte d'Irène d'abord, puis prise de conscience de la solitude par Sherlock et de la régression que représenterait, dans son

${ }^{39}$ Dans le pilote de la première saison, le rôle du second de Gregson était tenu par un inspecteur latino. Il est remplacé par le personnage de Marcus Bell dès le second épisode. On notera (effet de Canon) que le patronyme de Bell reprend celui de Joseph Bell, professeur de médecine à l'université d'Édimbourg, l'un des modèles avérés sur lequel Conan Doyle construisit Sherlock Holmes. 
processus de guérison, le départ de Joan au terme de son accompagnement dans le courant de la première saison. Dans la seconde, l'idylle naissante entre Joan et Mycroft accélère chez Sherlock le sentiment d'un risque de déséquilibre qu'entraînerait le départ de Joan, et les affrontements entre les deux frères ne masquent pas la jalousie de Sherlock.

Dès le début de la première saison, Sherlock évoque sa difficulté à supporter l'ennui. Dans un passage fameux du Canon, Watson voit dans ces moments où la passion intellectuelle vient à manquer, la raison du recours de Holmes à la cocaïne. Alors que Holmes explique à Irène Adler la destruction par l'addiction qui suivit sa disparition [Irène/The Woman, 1.23], il évoque un usage antérieur de la drogue purement festif. De cette nuance se déduit l'importance dans Elementary du thème de la relation à l'autre - le pair manquant évoqué plus haut - ainsi que du motif du soin. La série s'inscrit ici dans la suite des thérapies apportées à la pathologie de Holmes : chez Conan Doyle, le sauver de l'ennui que les textes présentent comme une forme de mélancolie par l'enquête et la passion de l'énigme; chez Nicholas Meyer, dans La Solution à $7 \% 4^{40}$, l'addiction de Holmes conduit Watson à le mener à Vienne consulter Freud; dans Elementary, la guérison ne peut venir que de l'amitié et de la prise en compte de l'autre. Reste alors à Holmes à dépasser son mépris pour les réunions des groupes de parole où Joan le contraint à aller, et à être en position de prodiguer à son tour le soin du parrainage pour accompagner lors de sa postcure un autre toxicomane.

La bienveillance de Joan relève ouvertement d'une éthique du care. La série souligne qu'elle est une remarquable chirurgienne dont le savoir est considérable: elle n'est pas une généraliste au talent moyen comme le Watson de Conan Doyle mais possède un talent qui, sans l'identifier au docteur House (lui-même construit comme une variation médicale du personnage de Holmes), la renverrait plutôt au personnage de Cristina Young, la brutalité arriviste en moins, dans la série Grey's Anatomy ${ }^{41}$ (ABC, 2005-). A partir du moment où Holmes comprend l'intérêt que Joan trouve aux enquêtes dans lesquelles elle l'accompagne et qu'il perçoit chez elle un authentique potentiel déductif, la série s'ouvre au thème de la formation de Joan par Sherlock et de la transmission de son savoir à un personnage qui, une fois l'apprentissage achevé, a alors vocation à se constituer en pair. Le thème de l'empathie et de l'ouverture progressive du solipsisme holmésien au monde et à l'autre devient alors ce fil scénaristique continu qui transcende la répétition des chutes où se boucle l'affaire examinée dans l'épisode. Les scénarios des épisodes fouillent cette

${ }^{40}$ Nicholas Meyer, The 7\% Solution, Norton, Londres, E.P. Dutton, 1974 : adapté au cinéma par Herbert Ross en 1976.

${ }^{41}$ Pour une lecture de l'éthique du care appliquée à la série télé Grey's anatomy, voir Laurent Jullier et Barbara Laborde, 'Grey’s anatomy', du cœur au care, Paris, PUF, 2012. 
critique de l'égoïsme, de la passion autocentrée de Holmes poussant, par exemple, l'arrogance et l'orgueil de Holmes jusqu'à les rendre directement responsables d'une grave blessure de Marcus Bell [2.10 ; Tremors].

Enfin, cette ouverture à l'autre s'exprime par une mise en scène de la parole et de la pédagogie de l'explication qui, progressivement dans la série, abandonne la théâtralité hautaine des cinq dernières minutes, sorte de fatalité de la chute policière, hypertrophiée par le ridicule, par exemple, du personnage d'Hercule Poirot ${ }^{42}$.

Ainsi, exaspérée par ce que les Sud-Américains appellent des sherlockmitos - ces déductions sidérantes avec lesquelles Holmes bluffe son monde - Joan lui rend, sur un mode comique absurde, la monnaie de sa pièce, forçant Holmes à reconnaître que, dans son rôle de mentor, le partage est nécessaire et la condescendance un obstacle.

SHERLOCK. Ils ont quitté les lieux avec 33 millions en petites coupures; ils ont chargé leur butin dans une ambulance fabriquée aux États-Unis à la fin des années 90 et sont partis depuis à peine plus d'une heure.

JOAN. Le chauffeur a un oil paresseux. Deux des voleurs se sont rencontrés en jouant au basket et le troisième souffre d'un lupus canin. Vous voyez ce que ça fait ! Expliquez-moi au moins.

SHERLOCK. Très bien. Venez.

[1.1, 26'; Tempête sous un crâne/Snow Angels]

\section{L'amateur au pays des experts}

À partir d'une variation pariant sur la distance et la différence, la décontextualisation produit un effet de commentaire sur les formes passées. Mais de ce processus s'ensuit nécessairement une recontextualisation qui, elle, implique de lire les contenus déplacés à l'intérieur de la synchronie qui l'accueille : en l'occurrence, le rapport d'Elementary avec le contexte général des séries policières. Cette dynamique permet un réengendrement sériel de la fiction dans Elementary qui ne se nourrit pas uniquement de l'inventivité du cas présenté à Holmes à chaque nouvel épisode. On a précédemment noté la tension existant entre deux types d'imaginaire du récit policier déterminant l'histoire même de ses formes : détection contre roman noir. Si elle exigerait d'être traitée pour elle-même, et considérablement nuancée, cette distinction fonctionnelle en recoupe bien d'autres: Europe/Amérique, détective amateur/police institutionnelle, enquête individuelle/équipe policière, primat du récit de la méthode/action policière, etc. Mais cette distinction prend un sens particulier dans le contexte qui domine les séries policières depuis les années 2000, depuis que l'imaginaire de Columbo (NBC, 1968-1978 et $\mathrm{ABC}, 1989-2003)$ l'a cédé à celui des Experts.

${ }^{42}$ Voir le final du Crime de l'Orient Express de Sidney Lumet (1974), avec Albert Finney dans le rôle d'Hercule Poirot. 
Les police procedurals ont, ces dernières années, substitué au règne des enquêteurs, au primat des filatures, des poursuites et des interrogatoires, aux enjeux de conflits entre flics et coupables, le spectacle d'une autorité nouvelle tirant sa force du caractère incontestable des preuves qu'elle fournit de façon scientifique : nous sommes désormais entrés dans le règne de la toute-puissance de la police scientifique (forensics), de la médecine médico-légale, de l'expertise et du profiling. Gérard Wacjman a brillamment montré dans un essai, Les Experts : la police des morts, tout l'enjeu idéologique et culturel de ce changement de paradigme imaginaire survenu avec le triomphe de la fiction généralisée de l'expertise ${ }^{43}$. Or, Sherlock Holmes n'est pas un expert... c'est un amateur.

Les Experts, série créée par Anthony Zuiker et diffusée depuis 2000 par CBS, est désormais le paradigme archi-dominant d'un discours qui assure pouvoir identifier avec certitude le coupable, quitte à délaisser la toujours problématique question du mobile, de la motivation et du sens du geste, au profit de l'objectivité des traces que produisent les corps dans leurs actions. «Symptôme éclatant » d'une culture fondée sur l'omniprésence du discours scientifique, la série en est une «arme communicationnelle majeure », selon Wacjman. Mais si, comme il l'écrit, « le scientifique et le médecin marchent côté à côte et entrent ensemble dans la police ${ }^{44}$, Holmes n'est précisément ni médecin ni scientifique : c'est un amateur - bien évidemment, pas au sens d'une compétente insuffisante mais, au contraire, au sens d'un savoir supérieur et mû par la passion intellectuelle - et qui tient à sa position de consultant pour des raisons, sur le fond, avant tout éthiques ; ainsi, précise-t-il dès le premier épisode :

SHERLOCK. Avant d'être collé en pension pour toxicos, je travaillais comme consultant à Scotland Yard.

JOAN. Vous étiez enquêteur?

SHERLOCK. Non, j'étais consultant. Je n'étais pas payé pour mes services et je n'avais à répondre de mes actes qu'à moi-même.

$$
\text { [1.1, 5', Sherlock et Watson/Pilot }]
$$

La gratuité de son conseil le distingue du consultant privé, appointé par les entreprises et les institutions, en ces temps de discours communicationnel généralisé45. La parole de Holmes, on le verra, est d'un tout autre ordre. Cette liberté face à la rémunération est d'emblée assortie d'une éthique de la responsabilité qui rattache le détective à l'artiste, l'esthète ou l'amateur qui ne prostitue pas son art et s'en trouve seul comptable et en ce sens, Sherlock au XXI ${ }^{\mathrm{e}}$ siècle conserve les

\footnotetext{
${ }^{43}$ Gérard Wacjman, Les Experts. La Police des morts, Paris, Puf, 2012.

${ }^{44}$ Wacjman, p. 63.

45 Par ailleurs, ce terme est canonique et renvoie à l'expression « consulting detective » chez Conan Doyle.
} 
traits constitutifs de sa part bohème et dandy présente dès l'origine de son mythe.

La substitution du médecin au détective, ou cette façon de faire du médecin un détective, marque en même temps une certaine défaite de la pensée. C'est-à-dire que, contre le détective homme de lettres, de fiction, c'est-à-dire pour qui «la vérité a structure de fiction », comme Lacan le dit pour opposer savoir et vérité46, l'arrivée du héros-médecin est l'indicateur que nous sommes entrés dans l'époque du savoir sûr, au temps de la certitude obligatoire $» 47$.

Or, Holmes est bien trop cabotin, acteur et metteur en scène de sa propre performance pour renoncer à la dramatisation du sens et de la solution. Il n'y a rien d'obligatoire dans la solution qui n'a rien de certain, tant elle dépend non pas du réglage des machines mais de la figure toujours héroïque de l'herméneute : Elementary, en ces temps d'expertise et de communication, conjure la défaite de la pensée que souligne Wacjman. Sherlock continue à tirer sa puissance de l'exercice de la pensée, de sa culture considérable, protéiforme et autodidacte et de la force analytique de sa parole: autant de dimensions qui reviennent à situer l'événement - et l'intérêt romanesque - du récit de détection dans la fascination pour l'intelligence, l'astuce, le wit, l'esprit, aussi pour le soupçon que sous la surface des signes résident d'autres récits. Un tel héroïsme de l'intelligence évite la réduction du jeu logique et policier à de purs protocoles ou comptes rendus expérimentaux.

Sherlock tatoué est la contre-mesure idéale à l'ordre de l'expertise déclinée aujourd'hui dans cette fiction néo-positiviste élaborée au seuil des années 2000 par Zuicker, et Elementary semble s'opposer terme à terme à la description que Wacjman, dans une comparaison au départ établie avec Columbo, propose de ce qui se joue dans Les Experts et ses dérivés (NCIS, Body of Proof [ABC, 20112013], Bones [FOX, 2005-], Rizzoli \& Isles [TNT, 2010-], Women's Murder club [ABC, 2007-2008], etc.) :

[...] Serait fini le temps de l'intuition, de la perspicacité, de l'imagination créatrice, de la déduction, de la réflexion parfois erratique, parfois fantasque, de la fulguration, finalement de l'intelligence et de la pensée [...]. Être soulagé, libéré, délivré du travail de penser, pourquoi pas ? Mais être du coup privé, sevré de la jouissance de penser est une tout autre affaire..$^{48}$

Or la génialité névrotique de Holmes en fait un monstre d'intelligence. Et les épisodes où l'on suit la passion de Holmes et d'Irène Adler montrent bien à quel point chez lui se confondent la jouissance intellectuelle et physique, la raison et l'émotion. Contre la

${ }^{46}$ Wacjman renvoie à J. Lacan, D'un Autre à l'autre, Le Séminaire - Livre XVI, Paris, Le Seuil, 2006, p. 388

${ }^{47}$ Wacjman, p. 63

${ }^{48}$ Wacjman, p. $88-89$ 
compétence froide et étroite de l'expert, l'amateur est une figure passionnée de la culture et de la pensée et qui est signifiée comme individualité hors-norme. Délirant, arrogant, hyperactif, mais aussi, dans la manière dont Elementary traite de son déséquilibre, de ses obsessions et de troubles comportementaux, Holmes est, à la fois, la figure de la fragilité humaine et de l'héroïsme de la pensée, toujours susceptible d'en remontrer aux formes appauvrissantes, sur le plan de l'imaginaire, de l'objectivisme et du rationalisme. La rationalité que sert Holmes dans Elementary, depuis le Dupin de Poe et la question de l'imagination, de l'abduction créatrice, de l'invention de la fiction logique qui permet de penser la solution à une énigme, est le contraire du scientisme, du positivisme et de la réduction du signe à l'illusion de la certitude. Contrairement à ce qu'écrivait Watson au début d'un «Scandale en Bohême » : «Je tiens Sherlock Holmes pour la machine à observer et à raisonner la plus parfaite qui ait existé sur la planète ${ }^{49}$. Sherlock au XXI ${ }^{\mathrm{e}}$ siècle n'est pas une machine intellectuelle.

La réécriture actualisée du mythe holmésien, dans Elementary, permet à Doherty de redistribuer des caractérisations connues du personnage et d'y trouver les facteurs d'une évolution significative. Mais il ne s'agit pas d'opposer de façon antithétique les vertiges de la pensée et la médiocrité de la vie, le solipsisme intellectuel et l'empathie, le passé Adler/le présent Joan : les deux dimensions sont intimement liées procédant l'une de l'autre et assurant la dynamique du renouvellement dans la série. À mesure que s'affirme toujours la nécessité de l'individualisme et de l'exception de la pensée, d'autres dimensions de l'existence deviennent de plus en plus accessibles pour Sherlock: l'inquiétude, la sollicitude, la capacité à reconnaître son erreur ou à féliciter l'autre, Joan, Bell ou Gregson. La victoire logique qui détermine la chute des récits policiers est alors le contre-champ d'un récit qui court sur l'ensemble des deux saisons et qui porte sur l'apprentissage que Sherlock fait des relations humaines. À l'enchaînement du traumatisme du deuil, de l'addiction et du soin, répond la construction d'une relation privilégiée avec Watson et que ne cessent de commenter les deux personnages de la série : cette relation, pour durer d'épisodes en saisons, trouve alors sa meilleure expression dans la structure de la transmission ou de l'apprentissage de la détection pour Watson, et dans l'horizon de son émancipation.

Si la parité prend du temps, celui de la formation pour se construire dans le domaine de la pensée déductive, c'est par la parole que Joan s'impose très rapidement, dès la première saison, comme une authentique interlocutrice pour Sherlock. Elementary est une série de la joute oratoire, du mot d'esprit, de la vitesse de la réplique ; dans un monde d'images, dans un jeu policier qui risque toujours de ne se

49 Arthur Conan Doyle, «Scandale en Bohême » dans Les Aventures de Sherlock Holmes, édition intégrale, Paris, NéO, tome 15, p. 15. 
réduire qu'à la traduction du signe en certitude, Sherlock joue encore de la parole, de la formule comique, hautaine, arrogante, de tous les ressorts de l'ironie critique, déstabilisant ou sidérant ses auditeurs par la qualité syntaxique, l'hyper-précision lexicale, les vertiges de l'érudition et la définition qui font de son discours une performance. La fulguration est séduction de l'intelligence déployée sous l'espèce du trait, de la formule, de la saillie : rien n'est plus étranger à Sherlock que l'esprit d'escalier, et les répliques fusent, mais elles ne sont jamais plus grisantes qu'en compagnie de partenaires à la hauteur. Gregson, Bell souvent, mais surtout Irène/Moriarty, Joan et Mycroft sont les esprits supérieurs avec lesquels le jeu/la joute méritent d'être joués.

Il n'y a pas de fable possible de l'intelligence et de l'émancipation qui ne passe par la conquête de son propre langage, par sa maitrise et la capacité de résistance à l'autorité d'autrui. La séduction du pilote aura été de ramener dans l'Amérique des années 2010, dans la ritualité des discours policiers et de leur stéréotype de commissariat, d'équipiers et de procédure, cette virtuosité de la parole - ou préciosité toute européenne du discours, c'est selon - incarnée par Sherlock. À charge pour les chutes de boucler les récits formulaires - the case is closed - sur la bonne formule - bon mot, paradoxe, suspension, la pointe ou le concetto d'un poème déductif et logique... , mais cela, tout en conservant à l'histoire d'une relation se constituant au long cours des saisons, une séduction pour les spectateurs qui transcende l'intérêt local des épisodes, et pour les personnages de Joan Watson et de Sherlock Holmes s'imposant comme un enjeu, personnel et profond, qui n'ait rien d'élémentaire.

\section{Bibliographie}

[Anonyme] http://www.audiencesusa.com/(dernière consultation : 15 juin 2015).

[Anonyme] http://www.leblogtvnews.com/article-bilan-d-audiencede-la-saison-1-d-elementary-sur-m6-122772623.html (dernière consultation : 15 juin 2015).

BARNes Alan, Sherlock Holmes on Screen, The Complete Film and TV History, Londres, Titan Books, 2011.

CARr Caleb, L'aliéniste (1996), Paris, Pocket, 2004.

CouEgnas Daniel, «L'effet Watson », Les cahiers de l'imaginaire, ${ }^{\circ} 14$.

DAY Thomas, L’instinct de l'équarisseur : vie et mort de Sherlock Holmes (Mnémos, 2002), Paris, coll. « Folio SF », Gallimard, 2004. 
DESMET Maud, Les Confessions silencieuses du cadavre : de la fiction d'autopsie aux figures des morts dans les séries et films policiers contemporains (1991-2013), soutenue en mars 2013 à l'université de Poitiers.

Doctorow E.L., Waterworks (1994), La Machine d'eau de Manhattan, Paris, Flammarion, 1996.

EISENZWEIG Uri, Le Récit impossible forme et sens du roman policier, Paris, Bourgois, 1986.

GenetTe Gérard, Palimpseste, la littérature au second degré, Paris, coll. « Poétique », Seuil, 1982.

GREEN Richard Lancelyn, éd., Letters to Sherlock Holmes, Londres, Penguin books, 1985 .

JULLIER Laurent, Barbara LABORDE, 'Grey's anatomy', du cour au care, Paris, PUF, 2012.

LEVET Natacha, Sherlock Holmes, de Baker Street au grand écran, Paris, Autrement, 2012.

MELLIER Denis, «L'Aventure de la faille apocryphe ou Reichenbach et la Sherlock-fiction ", in Sherlock Holmes et le signe de la fiction, éd. Denis Mellier, Fontenay Saint-Cloud, coll. «Signes », ENS éditions, 1999, p.164-166.

MEYER Nicholas, The 7\% Solution, Norton, Londres, E.P. Dutton, 1974.

Mryoshi Masao, The Divided Self: a Perspective on the Literature of the Victorians, New York, University Press, 1969.

MOFFAT Stephen, http://www.lemonde.fr/culture/article/2014/04/18/sherlock-holmesest-comme-james-bond-un-heros-moderne 4399794 3246.html (dernière consultation : 15 juin 2015).

Morel Max Philippe, Sherlock Holmes au cinéma et à la télévision, Strasbourg, Les Évadés de Dartmoor, 2013.

Reouven René, Histoires secrètes de Sherlock Holmes, Paris, Denoël, 1993. 
STOuT Rex, "Watson was a woman », The Saturday Review of Literature, $\mathrm{n}^{\circ} 1$, March, p. 3-4.

Thompson June, «Book Review», Sherlock Holmes Gazette, nº12, summer 1995, p.28.

Thompson June, Holmes and Watson : a Study in Friendship (1995), Paris, coll. « Le Masque » ${ }^{\circ}$ 2292, Librairie des Champs-Élysées, 1996.

Tracy Jack (éd.), Sherlock Holmes, the Published Apocrypha by Arthur Conan Doyle and Associated Hands, Boston, Houghton Mifflin Company, 1980.

TRUCHON Catherine, «Sherlock Holmes ou le surhomme détective », http://popenstock.ca/dossier/article/sherlock-holmes-ou-lesurhomme-detective (dernière consultation : 15 juin 2015).

WacJMan Gérard, Les Experts. La Police des morts, Paris, PUF, 2012.

Denis Mellier est professeur à l'université de Poitiers (France), où il enseigne la littérature générale et comparée ainsi que le cinéma. Il a publié de nombreux articles sur la fiction fantastique, l'horreur au cinéma, les esthétiques réflexives, et les relations entre la littérature policière et le roman contemporain. Parmi ses ouvrages, L'Ecriture de l'excès. Poétique de la terreur et fiction fantastique, Champion, 1999. Grand prix de l'imaginaire, catégorie «Essai» 2000 ; La Littérature fantastique, "Mémo », Seuil, 2000, 64 p. Grand prix de l'imaginaire, catégorie «Essai» 2000; Textes fantômes. Fantastique et autoréférence. Kimé, 2001; Les Écrans meurtriers. Essais sur les scènes spéculaires du thriller, éditions du CEFAL, Liège, 2001. Il a dirigé jusqu'en 2013 la publication de la revue Otrante, arts et littérature fantastique (éditions Kimé). Il travaille, ces derniers temps, plus particulièrement sur les rapports entre violence et représentation, les relations littérature et cinéma, les arts visuels (arts numériques et bande dessinée) et les questions de théorie littéraire (poétique comparée, culture médiatique et littérature, théorie de la fiction).

Denis Mellier is a professor at the University of Poitiers, France, where he teaches comparative and general literature and cinema. He has published many articles on the Fantastic, horror movies, the poetics of reflexivity and the relationships between crime fiction and general literature. Among his books, L'Ecriture de l'excès. Poétique de la terreur et fiction fantastique, Paris, Champion, 1999. He was awarded the Grand prix de l'imaginaire, catégorie "Essai» 2000 ; La Littérature fantastique, "Mémo », Paris, Seuil, 2000, 64 p. Grand prix de l'imaginaire, catégorie «Essai» 2000 ; Textes fantômes. Fantastique et autoréférence. Paris, Kimé, 2001; Les Écrans meurtriers. Essais sur les scènes spéculaires du thriller, Liège, éditions du CEFAL, Liège, 2001. Until 2013, he was the editor of the academic journal, Otrante, Arts et littérature fantastique (Kimé publishing). Lately, his works have focused on the relationships between violence and representation, literature and cinema, and visual arts (graphic novels and digital arts, and issues of literary theory such as comparative poetics, media culture and literature and theory of fiction). 